\title{
Estimation of Combining Ability and Gene Action Studies in Brinjal (Solanum melongena L.)
}

\author{
Madhusmita Dishri* and H.N. Mishra \\ Department of Vegetable Science, Orissa University of Agriculture and Technology, \\ Bhubaneswar-751003, Odisha, India \\ *Corresponding author
}

Keywords

Brinjal, GCA, SCA, Yield, Gene action.

Article Info

Accepted:

28 September 2017

Available Online:

10 October 2017

\section{A B S T R A C T}

The present investigation was carried out to estimate GCA of eight parents and SCA of 28 crosses for ten quantitative traits in $8 \times 8$ half diallel mating design excluding reciprocals in brinjal. Experimental results revealed that among the parents, BBSR 202, BB 45C and BB 26 were found best on the basis of GCA effects while the crosses BBSR $202 \times$ BB 13, BB $26 \times$ BBSR 195-1 and BB 45C $\times$ BB 13 were found best on the basis of SCA effects for yield and contributing traits hence can be further exploited for commercial cultivation after multi-location testing. Further, the gene action studies revealed that the magnitude of $\sigma^{2} s$ were higher than $\sigma^{2} s$ for all the traits indicating pre-ponderant non additive gene action. Thus hybrid vigour could better be exploited for these traits

\section{Introduction}

Brinjal or eggplant is an important solanaceous vegetable crop of Indian origin having wide variability with respect to different qualitative characters. In India, it is one of the most common, popular and principal vegetable crops grown throughout the country except higher altitudes. Being a centre of origin, eggplant has a huge genetics in India which offers much scope for improvement through heterosis breeding (Venketanaresh et al., 2014). To cope up with ever increasing demand of brinjal, it has become very important to breed high yielding and suitable varieties to boost up the production per unit area per unit time (Joshi et al., 2008).
Now a day's development of new variety/hybrid with specific fruit characteristic is most desirable traits for the breeders of brinjal in India as well as in the world (Ansari and Singh, 2014). For an effective breeding programme in brinjal, information regarding the general and specific combining ability and the types of gene effects influencing various traits enables the plant breeder to evaluate parental material and to decide a suitable breeding procedure for maximum character improvement. The variance of general combining ability (GCA) and specific combining ability (SCA) are related to the type of gene action involved and the relative magnitudes of additive genetic 
variance, dominance variance and epistatsis in a breeding population. Thus the present investigation was undertaken to study GCA of the selected parental lines and SCA of crosses as well as gene action of different quantitative traits for formulating a sound breeding programme in brinjal.

\section{Materials and Methods}

The experiment was carried out at All India Coordinated Research Project on Vegetable Crops, Orissa University of Agriculture and Technology, Bhubaneswar, comprised of eight parental lines namely BB26, BB68, BB67, BB45C, BBSR195-1, BBSR202, BB13 and BB 44 (OUAT,BBSR) were crossed in half diallel mating design to get 28 $\mathrm{F}_{1}$ hybrids. These $28 \mathrm{~F}_{1}$ hybrids and eight parents were evaluated in randomized block design with three replications during Rabi season 2014. Each plant was transplanted at a distance of $60 \mathrm{~cm}$ between rows and $45 \mathrm{~cm}$ between plants. Standard agronomic practices were followed to raise the crop. Observations were recorded on five randomly selected plants from each entry over the replications for all the traits under study viz. days to first flowering, plant height $(\mathrm{cm})$, number of flowers per cluster, number of branches per plant, fruit length $(\mathrm{cm})$, fruit breadth $(\mathrm{cm})$, number of fruits per cluster, number of fruits per plant, average fruit weight (g), fruit yield per plant $(\mathrm{g})$. The data were subjected to appropriate statistical analysis. The combining ability estimates were calculated according to the Griffing's (1956) method 2 model I.

\section{Results and Discussion}

The variance due to general combining ability (gca) of parents and specific combining ability (sca) of crosses was significant for all the traits under study (Tables 1 and 2). The variance for GCA includes additive nature.
The perusal of GCA effects of the parents as presented in Table 1 revealed that none of the single parent has exhibited significant GCA effects for all the traits indicating differences in genetic variability for different traits among the parents.

Parent BB 45C showed maximum gca effects for earliness (days to first flowering). For fruit yield per plant lines BBSR 202, BB 45C and BB 26 exhibited highest positive GCA effects. All of these lines were also found good combiners for average fruit weight and number of fruits per plant. Besides this, BBSR 195-1 exhibited maximum significant GCA effects for fruit length, fruit breadth and plant height. BB 13 for number of fruits per cluster and BB 44 for number branches per plant registered highest significant GCA effects. These observations revealed that yield could be improved by using these parents in hybridization programmes for the accumulation of favourable genes. Significant positive GCA effects for yield and yield contributing traits in brinjal were also reported by Rai and Asati (2011), Kamalakkannan et al., (2007) and Thangavel (2011).

The sca effects and per se performance of crosses are presented in Table 4. To confirm whether the crosses selected on the basis of sca effects were really the best performing ones, the five best crosses based on their mean performance and sca effects were selected. Variance for SCA includes nonadditive nature of total variance arising largely from dominance and epistatic deviations and are non-fixable in nature. Such non-fixable components are potential parameters for heterosis breeding which is very much useful in brinjal where commercial exploitation of heterosis is feasible. The estimates of sca effects of crosses pertaining to different characters are presented in Table 3 . 
Table.1 Estimates of general combining effects of parents for different traits in Brinjal

\begin{tabular}{|c|c|c|c|c|c|c|c|c|c|c|}
\hline Parent & $\begin{array}{l}\text { Days to } \\
\text { first } \\
\text { flowering }\end{array}$ & $\begin{array}{l}\text { Plant } \\
\text { height } \\
(\mathrm{cm})\end{array}$ & $\begin{array}{l}\text { Number of } \\
\text { flowers } \\
\text { per cluster }\end{array}$ & $\begin{array}{l}\text { Number of } \\
\text { branches } \\
\text { per plant }\end{array}$ & $\begin{array}{l}\text { Fruit } \\
\text { length } \\
(\mathrm{cm})\end{array}$ & $\begin{array}{l}\text { Fruit } \\
\text { breadth } \\
(\mathrm{cm})\end{array}$ & $\begin{array}{l}\text { Number of } \\
\text { fruits per } \\
\text { cluster }\end{array}$ & $\begin{array}{l}\text { Number } \\
\text { of fruits } \\
\text { per Plant }\end{array}$ & $\begin{array}{l}\text { Average } \\
\text { fruit } \\
\text { weight (g) }\end{array}$ & $\begin{array}{l}\text { Fruit yield } \\
\text { per plant (g) }\end{array}$ \\
\hline BB26 & 1.27 & $3.94 * *$ & -0.04 & 0.02 & $-2.28 * *$ & -0.17 & $-0.40 * *$ & -0.39 & $9.99 * *$ & $72.3 * *$ \\
\hline BB68 & 2.09 & 0.94 & $-0.61 * *$ & 0.00 & $0.84 *$ & -0.29 & $-0.17 * *$ & -0.53 & -0.28 & $-55.78^{*}$ \\
\hline BB67 & 0.22 & $-5.75 * *$ & $0.37 * *$ & -0.01 & $-1.05 * *$ & -0.75 & $-0.14 * *$ & $-.98 * *$ & $2.10 *$ & $-103.01 *$ \\
\hline BB45C & $-4.71 * *$ & 2.69 & $0.25 * *$ & 0.09 & $0.75 *$ & -0.38 & $0.19 * *$ & $2.09 * *$ & $-4.04 * *$ & $80.79 * *$ \\
\hline BBSR195-1 & -0.39 & $4.49 * *$ & -0.08 & -0.02 & $1.08 * *$ & $2.51 * *$ & $0.15 * *$ & 0.05 & $-6.45^{* *}$ & -37.99 \\
\hline BBSR202 & 1.91 & $-3.63 *$ & $0.35 * *$ & -0.14 & $0.69 *$ & 0.64 & 0.01 & $1.57 * *$ & $-3.95 * *$ & $87.17 * *$ \\
\hline BB13 & 0.06 & $-4.68 * *$ & $0.18 * *$ & $-0.36 * *$ & -0.36 & $-1.07 *$ & $0.24 * *$ & $-0.65^{*}$ & $3.61 * *$ & $5.63 * *$ \\
\hline BB 44 & -0.44 & 2.00 & $-0.43^{* *}$ & $0.42 * *$ & 0.34 & -0.49 & $0.11 *$ & $-1.15^{* *}$ & $3.21 * *$ & $-49.12 *$ \\
\hline SE (Gi) & 1.30 & 1.37 & 0.07 & 0.09 & 0.33 & 0.45 & 0.05 & 0.32 & 0.98 & 23.35 \\
\hline
\end{tabular}

Table.2 Estimates of specific combining effects of hybrids for different traits in Brinjal

\begin{tabular}{|c|c|c|c|c|c|c|c|c|c|c|}
\hline & $\begin{array}{l}\text { Days to } \\
\text { first } \\
\text { flowering }\end{array}$ & $\begin{array}{l}\text { Plant } \\
\text { height } \\
\text { (cm) }\end{array}$ & $\begin{array}{l}\text { Number } \\
\text { of flowers } \\
\text { per } \\
\text { cluster }\end{array}$ & $\begin{array}{l}\text { Number } \\
\text { of } \\
\text { branches } \\
\text { per Plant }\end{array}$ & $\begin{array}{l}\text { Fruit } \\
\text { length } \\
(\mathrm{cm})\end{array}$ & $\begin{array}{l}\text { Fruit } \\
\text { breadth } \\
(\mathrm{cm})\end{array}$ & $\begin{array}{l}\text { Number of } \\
\text { fruits per } \\
\text { cluster }\end{array}$ & $\begin{array}{l}\text { Number of } \\
\text { fruits per } \\
\text { Plant }\end{array}$ & $\begin{array}{l}\text { Average } \\
\text { fruit } \\
\text { weight (g) }\end{array}$ & $\begin{array}{l}\text { Fruit } \\
\text { yield per } \\
\text { plant (g) }\end{array}$ \\
\hline BB26×BB68 & 6.06 & 6.00 & $-1.11 * *$ & $1.19 * *$ & 0.04 & 0.66 & -0.03 & $3.55 * *$ & $-17.34 * *$ & 79.85 \\
\hline BB26×BB67 & -7.28 & $10.22^{*}$ & $1.29 * *$ & -0.08 & 0.36 & -0.79 & 0.14 & 0.82 & $6.70^{*}$ & $149.59 *$ \\
\hline BB26×BB45C & 4.46 & -1.45 & -0.24 & $-0.65^{*}$ & -0.28 & $-3.15^{*}$ & 0.01 & $-2.23 *$ & $16.21 * *$ & 44.06 \\
\hline BB26×BBSR195-1 & -3.86 & $-8.65^{*}$ & 0.40 & $0.57 *$ & $-2.34 *$ & -1.92 & -0.15 & 1.75 & $6.01 *$ & $190.81 *$ \\
\hline BB26 $\times$ BBSR202 & -3.16 & 4.67 & 0.10 & $-0.64 *$ & $-2.18 *$ & $3.81 *$ & -0.11 & $-2.43^{*}$ & $-15.89 * *$ & -324.56 \\
\hline BB26×BB13 & 5.62 & 4.22 & $0.42 *$ & $-0.65^{*}$ & 1.15 & 0.05 & -0.14 & -0.99 & $27.14 * *$ & $144.01 *$ \\
\hline BB26×BB 44 & $-8.81^{*}$ & -6.66 & $0.47 *$ & -0.03 & -0.01 & $-3.69 *$ & -0.11 & -1.44 & $16.85 * *$ & 11.22 \\
\hline BB68×BB67 & -0.29 & 3.09 & 0.07 & -0.09 & 1.80 & $-4.95 * *$ & $0.91 * *$ & $-2.81 * *$ & $34.59 * *$ & 62.70 \\
\hline BB68×BB45C & $-21.36 * *$ & $-12.85 * *$ & 0.04 & 0.12 & $-5.46 * *$ & 2.21 & -0.12 & 1.89 & $-10.48 * *$ & 20.90 \\
\hline BB68×BBSR195-1 & -0.83 & $-10.65^{*}$ & 0.25 & -0.36 & 0.74 & -1.82 & $0.42 * *$ & -1.86 & $6.30 *$ & -57.67 \\
\hline BB68×BBSR202 & $-12.98 * *$ & 4.07 & $1.24 * *$ & $-0.86 * *$ & $3.76 * *$ & -2.26 & -0.04 & 1.58 & $6.87 *$ & 87.14 \\
\hline
\end{tabular}




\begin{tabular}{|c|c|c|c|c|c|c|c|c|c|c|}
\hline BB68×BB13 & 6.87 & 5.92 & 0.00 & 0.27 & $-0.28 * *$ & $-3.96 * *$ & $-0.37 *$ & 0.63 & $9.62 * *$ & $149.40 *$ \\
\hline BB68×BB 44 & -2.63 & -4.96 & $0.47 *$ & 0.45 & $3.17 * *$ & -1.29 & $-0.44 * *$ & -0.67 & $-7.58^{*}$ & -85.25 \\
\hline BB67×BB45C & -4.50 & 1.54 & -0.21 & -0.19 & $3.07 * *$ & 1.54 & $-0.65 * *$ & $4.56^{* *}$ & $-17.93 * *$ & 30.14 \\
\hline BB67×BBSR195-1 & $-11.81 * *$ & 3.64 & $0.47 *$ & $-1.10 * *$ & -1.98 & -0.17 & 0.09 & -1.95 & $6.81 *$ & -58.92 \\
\hline BB67×BB13 & -3.27 & $-10.29 *$ & $-0.52 *$ & 0.04 & $-2.50 *$ & -0.26 & -0.10 & -0.29 & $-20.51 * *$ & $-191.83 *$ \\
\hline BB67×BB 44 & -5.73 & -1.17 & $-0.46^{*}$ & 0.25 & 0.51 & 2.80 & -0.07 & -0.46 & 0.34 & -5.34 \\
\hline BB45C $\times$ BBSR195-1 & 3.12 & 0.00 & $0.77 * *$ & 0.31 & $5.95 * *$ & $4.81 * *$ & $-0.34 *$ & 0.47 & -5.86 & -65.23 \\
\hline BB45C $\times$ BBSR202 & -2.08 & $17.52 * *$ & -0.18 & -0.44 & $4.05 * *$ & -0.46 & 0.10 & -0.16 & 4.52 & 49.42 \\
\hline BB45C $\times$ BB13 & -5.70 & $22.17 * *$ & $1.30 * *$ & 0.02 & $2.51 *$ & -1.75 & $-0.53 * *$ & -1.87 & $28.71 * *$ & $186.73 *$ \\
\hline BB45C $\times$ BB 44 & 4.17 & $21.39 * *$ & $0.60 * *$ & 0.37 & -1.06 & -1.20 & $-0.70 * *$ & 1.37 & -3.81 & 86.32 \\
\hline BBSR195-1×BBSR202 & -5.64 & 5.72 & $1.11 * *$ & $-0.84 * *$ & -1.81 & 0.67 & $-0.76^{* *}$ & -1.00 & $-7.14^{*}$ & -18.10 \\
\hline BBSR195-1×BB13 & -2.65 & $13.17 * *$ & 0.13 & $-0.75^{* *}$ & $-2.30 *$ & $3.60 *$ & $-0.69 * *$ & -0.04 & $-20.91 * *$ & $-228.17 *$ \\
\hline BBSR195-1×BB 44 & -2.08 & $-14.51 * *$ & -0.03 & $-0.83 * *$ & -0.92 & $3.86 * *$ & $-0.36^{*}$ & 1.89 & -1.31 & 103.04 \\
\hline BBSR202×BB13 & -4.96 & -0.61 & 0.36 & 0.45 & -1.37 & 1.84 & $-0.45 * *$ & $4.29 * *$ & $6.00 *$ & $357.69 *$ \\
\hline BBSR202× BB 44 & -1.46 & 1.81 & $-0.88 * *$ & $0.57 *$ & -1.21 & -1.49 & $1.78 * *$ & 1.03 & -3.98 & 16.11 \\
\hline BB13×BB 44 & -0.67 & $-15.08 * *$ & 0.21 & 0.49 & -0.29 & $7.90 * *$ & $1.18 * *$ & 1.48 & 3.82 & 140.41 \\
\hline SE (Sij) & 3.99 & 4.21 & 0.20 & 0.27 & 1.02 & 1.38 & 0.14 & 0.99 & 2.99 & 71.58 \\
\hline
\end{tabular}

Table.3 Combining ability variance for various traits in Brinjal

\begin{tabular}{|c|c|c|c|c|c|c|c|c|c|c|}
\hline $\begin{array}{l}\text { Source of } \\
\text { variation }\end{array}$ & $\begin{array}{l}\text { Days to } \\
\text { first } \\
\text { flowering }\end{array}$ & $\begin{array}{l}\text { Plant } \\
\text { height } \\
(\mathrm{cm})\end{array}$ & $\begin{array}{l}\text { Number } \\
\text { of flowers } \\
\text { per } \\
\text { cluster }\end{array}$ & $\begin{array}{l}\text { Number of } \\
\text { branches } \\
\text { per plant }\end{array}$ & $\begin{array}{l}\text { Fruit } \\
\text { length } \\
\text { (cm) }\end{array}$ & $\begin{array}{l}\text { Fruit } \\
\text { breadth } \\
(\mathrm{cm})\end{array}$ & $\begin{array}{l}\text { Number } \\
\text { of fruits } \\
\text { per } \\
\text { cluster }\end{array}$ & $\begin{array}{l}\text { Number } \\
\text { of fruits } \\
\text { per plant }\end{array}$ & $\begin{array}{l}\text { Fruit } \\
\text { weight } \\
(\mathrm{g})\end{array}$ & $\begin{array}{l}\text { Fruit } \\
\text { yield per } \\
\text { plant }(g)\end{array}$ \\
\hline$\sigma^{2} g$ & 2.67 & 14.42 & 0.13 & 0.04 & 1.23 & 1.04 & 0.04 & 3.92 & 82.10 & \begin{tabular}{|c|}
13973.55 \\
\end{tabular} \\
\hline$\sigma^{2} s$ & 65.38 & 112.51 & 0.55 & 0.38 & 5.65 & 9.19 & 0.39 & 9.17 & 681.13 & 58822.52 \\
\hline$\sigma^{2} g / \sigma^{2} S$ & 0.04 & 0.13 & 0.23 & 0.10 & 0.22 & 0.11 & 0.12 & 0.43 & 0.12 & 0.24 \\
\hline
\end{tabular}


Table.4 Ranking of best five parents, crosses based on per se performance, sca and gca effects with respect to ten quantitative traits

\begin{tabular}{|c|c|c|c|c|c|c|}
\hline \multirow[t]{2}{*}{ Traits } & \multirow[t]{2}{*}{$\begin{array}{l}\text { Best general } \\
\text { combiner }\end{array}$} & \multirow[t]{2}{*}{ Best parent } & \multirow[t]{2}{*}{$\begin{array}{l}\text { Best crosses on the basis of } \\
\text { mean }\end{array}$} & \multirow[t]{2}{*}{ Best crosses on the basis of sca } & \multicolumn{2}{|c|}{$\begin{array}{l}\text { GCA effect of } \\
\text { parents }\end{array}$} \\
\hline & & & & & P1 & $\mathbf{P 2}$ \\
\hline $\begin{array}{l}\text { Days to } \\
\text { first } \\
\text { flowering }\end{array}$ & BB 45C(-4.71) & $\begin{array}{l}\text { BB45C }(95.50) \\
\text { BB13(96.50) } \\
\text { BB67(98.93) } \\
\text { BB26(100.00) } \\
\text { BBSR 202(100.99) }\end{array}$ & $\begin{array}{l}\mathrm{BB} 68 \times \mathrm{BB} 45 \mathrm{C}(70.00) \\
\mathrm{BB} 67 \times \mathrm{BBSR} 195-1(82.01) \\
\mathrm{BB} 45 \mathrm{C} \times \mathrm{BB} 13(83.63) \\
\mathrm{BB} 68 \times \mathrm{BBSR} 202(85.00) \\
\mathrm{BB} 67 \times \mathrm{BB} 45 \mathrm{C}(85.00)\end{array}$ & $\begin{array}{l}\text { BB } 68 \times \text { BB 45C }(-21.36) \\
\text { BB } 68 \times \text { BBSR 202(-12.98) } \\
\text { BB } 67 \times \text { BBSR 195-1(-11.81) } \\
\text { BB } 26 \times \text { BB 44(-8.81) } \\
\text { BB26 } \times \text { BB 67(-7.28) }\end{array}$ & $\begin{array}{l}\text { Low } \\
\text { Low } \\
\text { Low } \\
\text { Low } \\
\text { Low }\end{array}$ & $\begin{array}{l}\text { High } \\
\text { High } \\
\text { Low } \\
\text { Low } \\
\text { Low }\end{array}$ \\
\hline $\begin{array}{l}\text { Number } \\
\text { of flowers } \\
\text { per } \\
\text { cluster }\end{array}$ & $\begin{array}{l}\text { BB 67(.37) } \\
\text { BBSR 202(.35) } \\
\text { BB 45C (.25) } \\
\text { BB 13(.18) }\end{array}$ & $\begin{array}{l}\text { BB67(3.95) } \\
\text { BBSR 202(3.35) } \\
\text { BB45C (2.9) } \\
\text { BB13(2.86) } \\
\text { BB26(2.7) } \\
\end{array}$ & $\begin{array}{l}\text { BB45C ×BB13(5.18) } \\
\text { BB26 ×BB67(5.07) } \\
\text { BBSR 195-1 ×BBSR 202(4.82) } \\
\text { BB68 ×BBSR 202(4.42) } \\
\text { BB45C ×BBSR 195-1(4.38) } \\
\end{array}$ & $\begin{array}{l}\text { BB 45C × BB 13(1.30) } \\
\text { BB26 × BB 67(1.29) } \\
\text { BB } 68 \times \text { BBSR 202(1.24) } \\
\text { BBSR 195-1 × BBSR 202(1.11) } \\
\text { BB 45C × BBSR195-1(0.77) } \\
\end{array}$ & $\begin{array}{l}\text { High } \\
\text { Low } \\
\text { Low } \\
\text { Low } \\
\text { High }\end{array}$ & $\begin{array}{l}\text { High } \\
\text { High } \\
\text { High } \\
\text { High } \\
\text { Low }\end{array}$ \\
\hline $\begin{array}{l}\text { Plant } \\
\text { height } \\
(\mathrm{cm})\end{array}$ & $\begin{array}{l}\text { BBSR 195-1(4.49) } \\
\text { BB 26(3.94) }\end{array}$ & $\begin{array}{l}\text { BBSR 195-1(109.65) } \\
\text { BB44(108.60) } \\
\text { BB 68(101.58) } \\
\text { BB26(98.73) } \\
\text { BB67(83.57) } \\
\end{array}$ & $\begin{array}{l}\mathrm{BB} 45 \mathrm{C} \times \mathrm{BB} 44(121.10) \\
\mathrm{BB} 45 \mathrm{C} \times \mathrm{BB} 13(115.20) \\
\mathrm{BB} 45 \mathrm{C} \times \mathrm{BBSR} 202(111.60) \\
\mathrm{BBSR} 195-1 \times \mathrm{BB} 13(108.00) \\
\mathrm{BB} 26 \times \mathrm{BB} 68(105.90)\end{array}$ & $\begin{array}{l}\text { BB 45C } \times \text { BB 13(22.17) } \\
\text { BB 45C } \times \text { BB 44(21.39) } \\
\text { BB 45C } \times \text { BBSR 202(17.52) } \\
\text { BBSR 195-1 × BB 13(13.17) } \\
\text { BB 26× BB 67(10.22) }\end{array}$ & $\begin{array}{l}\text { Low } \\
\text { Low } \\
\text { Low } \\
\text { High } \\
\text { High }\end{array}$ & $\begin{array}{l}\text { Low } \\
\text { Low } \\
\text { Low } \\
\text { Low } \\
\text { Low }\end{array}$ \\
\hline $\begin{array}{l}\text { Number } \\
\text { of } \\
\text { branches } \\
\text { per plant }\end{array}$ & BB 44(.42) & $\begin{array}{l}\text { BBSR 195-1(6.7) } \\
\text { BBSR 202(6.02) } \\
\text { BB67(5.98) } \\
\text { BB45C }(5.65) \\
\text { BB44(5.45) } \\
\end{array}$ & $\begin{array}{l}\mathrm{BB} 26 \times \mathrm{BB} 68(6.46) \\
\mathrm{BB} 68 \times \mathrm{BB} 44(6.12) \\
\mathrm{BB} 45 \mathrm{C} \times \mathrm{BB} 44(6.12) \\
\mathrm{BBSR} 202 \times \mathrm{BB} 44(6.10) \\
\mathrm{BB} 67 \times \mathrm{BB} 44(5.91) \\
\end{array}$ & $\begin{array}{l}\text { BB26 × BB 68(1.19) } \\
\text { BBSR } 202 \times \text { BB } 44(0.57)\end{array}$ & Low & Low \\
\hline $\begin{array}{l}\text { Fruit } \\
\text { length } \\
(\mathrm{cm})\end{array}$ & $\begin{array}{l}\text { BBSR 195-1(1.08) } \\
\text { BB 68(.84) } \\
\text { BB 45C }(.75)\end{array}$ & $\begin{array}{l}\text { BBSR 195-1(16.73) } \\
\text { BB13(14.06) } \\
\text { BB44(13.83) }\end{array}$ & $\begin{array}{l}\text { BB45C } \times \text { BBSR 195-1(21.02) } \\
\text { BB45C ×BBSR 202(18.73) } \\
\text { BB68 ×BBSR202(18.53) }\end{array}$ & $\begin{array}{l}\text { BB 45C } \times \text { BBSR 195-1(5.95) } \\
\text { BB 45C } \times \text { BBSR 202(4.05) } \\
\text { BB } 68 \times \text { BBSR 202(3.76) }\end{array}$ & $\begin{array}{l}\text { High } \\
\text { High } \\
\text { High }\end{array}$ & $\begin{array}{l}\text { High } \\
\text { High } \\
\text { High }\end{array}$ \\
\hline
\end{tabular}




\begin{tabular}{|c|c|c|c|c|c|c|}
\hline & BBSR 202(.69) & $\begin{array}{l}\text { BBSR 202(13.57) } \\
\text { BB68(13.04) }\end{array}$ & $\begin{array}{l}\text { BB68 ×BB 44(17.59) } \\
\text { BB45C ×BB13(16.14) }\end{array}$ & $\begin{array}{l}\text { BB68 × BB 44(3.17) } \\
\text { BB 67× BB 45C (3.07) }\end{array}$ & $\begin{array}{l}\text { High } \\
\text { High }\end{array}$ & $\begin{array}{l}\text { Low } \\
\text { High }\end{array}$ \\
\hline $\begin{array}{l}\text { Fruit } \\
\text { breadth } \\
(\mathbf{c m})\end{array}$ & BBSR 195-1(2.51) & $\begin{array}{l}\text { BB68 }(23.36) \\
\text { BB26(20.41) } \\
\text { BBSR 195-1(18.72) } \\
\text { BBSR 202(17.92) } \\
\text { BB67(17.12) }\end{array}$ & $\begin{array}{l}\text { BB45C ×BBSR 195-1(25.16) } \\
\text { BB13 ×BB44(24.55) } \\
\text { BBSR 195-1 ×BB44(24.10) } \\
\text { BBSR 195-1 ×BB13(23.26) } \\
\text { BB26 ×BBSR 202(22.50) }\end{array}$ & $\begin{array}{l}\text { BB } 13 \times \text { BB 44(7.90) } \\
\text { BB 45C × BBSR 195-1(4.81) } \\
\text { BBSR 195-1 × BB 44(3.86) } \\
\text { BB } 26 \times \text { BBSR 202(3.81) } \\
\text { BBSR 195-1 × BB 13(3.60) }\end{array}$ & $\begin{array}{l}\text { Low } \\
\text { High } \\
\text { High } \\
\text { Low } \\
\text { High }\end{array}$ & $\begin{array}{l}\text { Low } \\
\text { Low } \\
\text { Low } \\
\text { Low } \\
\text { Low }\end{array}$ \\
\hline $\begin{array}{l}\text { Number } \\
\text { of fruits } \\
\text { per } \\
\text { cluster }\end{array}$ & $\begin{array}{l}\text { BB13(.24) } \\
\text { BB 45C }(.19) \\
\text { BBSR 195-1(.15) }\end{array}$ & $\begin{array}{l}\text { BB45C }(3.10) \\
\text { BBSR 195-1(2.80) } \\
\text { BB13(2.62) } \\
\text { BBSR202(1.40) } \\
\text { BB44(1.2) }\end{array}$ & $\begin{array}{l}\mathrm{BBSR} 202 \times \mathrm{BB} 44(3.5) \\
\mathrm{BB} 13 \times \mathrm{BB} 44(3.13) \\
\mathrm{BB} 68 \times \mathrm{BB} 67(2.2) \\
\mathrm{BB} 68 \times \mathrm{BBSR} 195-1() \\
\mathrm{BB} 45 \mathrm{C} \times \mathrm{BBSR} 202(1.9)\end{array}$ & $\begin{array}{l}\mathrm{BBSR} 202 \times \mathrm{BB} 44(1.78) \\
\mathrm{BB} 13 \times \mathrm{BB} 44(1.18) \\
\mathrm{BB} 68 \times \mathrm{BB} 67(0.91) \\
\mathrm{BB} 68 \times \mathrm{BBSR} 195-1(0.42)\end{array}$ & $\begin{array}{l}\text { Low } \\
\text { High } \\
\text { Low } \\
\text { Low }\end{array}$ & $\begin{array}{l}\text { High } \\
\text { High } \\
\text { Low } \\
\text { High }\end{array}$ \\
\hline $\begin{array}{l}\text { Number } \\
\text { of fruits } \\
\text { per plant }\end{array}$ & $\begin{array}{l}\text { BB 45C (2.09) } \\
\text { BBSR 202(1.57) }\end{array}$ & $\begin{array}{l}\text { BB45C }(12.70) \\
\text { BBSR 202(12.68) } \\
\text { BBSR 195-1(11.05) } \\
\text { BB26(10.29) } \\
\text { BB67(9.29) }\end{array}$ & $\begin{array}{l}\text { BB } 67 \times \text { BB 45C }(16.25) \\
\text { BBSR } 202 \times \text { BB 13(15.8) } \\
\text { BB 45C ×BBSR 202(14.1) } \\
\text { BB68 ×BB 45C (14.05) } \\
\text { BB26 ×BB68(13.22) }\end{array}$ & $\begin{array}{l}\text { BB } 67 \times \text { BB } 45 C(4.56) \\
\text { BBSR } 202 \times \text { BB 13(4.29) } \\
\text { BB } 26 \times \text { BB 68(3.55) }\end{array}$ & $\begin{array}{l}\text { Low } \\
\text { High } \\
\text { Low }\end{array}$ & $\begin{array}{l}\text { High } \\
\text { Low } \\
\text { Low }\end{array}$ \\
\hline $\begin{array}{l}\text { Fruit } \\
\text { weight } \\
\text { (g) }\end{array}$ & $\begin{array}{l}\text { BB 26(9.99) } \\
\text { BB 13(3.61) } \\
\text { BB 44(3.21) }\end{array}$ & $\begin{array}{l}\text { BB 44(74.21) } \\
\text { BB26(70.10) } \\
\text { BBSR 202(68.20) } \\
\text { BBSR 195-1(65.10) } \\
\text { BB 67(62.10) }\end{array}$ & $\begin{array}{l}\text { BB26 ×BB13(110.70) } \\
\text { BB68 ×BB67(102.60) } \\
\text { BB26 ×BB44 }(100.00) \\
\text { BB45C ×BB13(98.24) } \\
\text { BB26 ×BB 67(84.54) }\end{array}$ & $\begin{array}{l}\text { BB68 × BB 67(34.59) } \\
\text { BB 45C } \times \text { BB 13(28.71) } \\
\text { BB26 } \times \text { BB 13(27.14) } \\
\text { BB26 } \times \text { BB 44(16.85) } \\
\text { BB26 } \times \text { BB 45C }(16.21)\end{array}$ & $\begin{array}{l}\text { Low } \\
\text { Low } \\
\text { High } \\
\text { High } \\
\text { High }\end{array}$ & $\begin{array}{l}\text { High } \\
\text { High } \\
\text { High } \\
\text { High } \\
\text { Low }\end{array}$ \\
\hline $\begin{array}{l}\text { Fruit } \\
\text { yield per } \\
\text { plant (g) }\end{array}$ & $\begin{array}{l}\text { BBSR 202(87.17) } \\
\text { BB 45C }(80.79) \\
\text { BB 26(72.3) }\end{array}$ & $\begin{array}{l}\text { BBSR 202(868.56) } \\
\text { BB26(724.66) } \\
\text { BBSR 195-1(718.70) } \\
\text { BB45C(712.95) } \\
\text { BB 67(578.91) }\end{array}$ & $\begin{array}{l}\text { BBSR202 ×BB13(1178.07) } \\
\text { BB45C ×BB13(1000.00) } \\
\text { BB26 ×BBSR 195-1(952.70) } \\
\text { BB26 ×BB13 }(949.60) \\
\text { BB45C ×BBSR 202(944.95) }\end{array}$ & $\begin{array}{l}\text { BBSR } 202 \times \text { BB 13(357.69) } \\
\text { BB } 26 \times \text { BBSR 195-1(190.8) } \\
\text { BB 45C × BB 13(186.73) } \\
\text { BB } 26 \times \text { BB 67(149.59) } \\
\text { BB } 68 \times \text { BB 13(149.40) }\end{array}$ & $\begin{array}{l}\text { High } \\
\text { High } \\
\text { High } \\
\text { High } \\
\text { Low }\end{array}$ & $\begin{array}{l}\text { High } \\
\text { Low } \\
\text { High } \\
\text { Low } \\
\text { High }\end{array}$ \\
\hline
\end{tabular}


Out of 28 crosses, significant sca effects in favourable direction were exhibited by 4 crosses for days to first flowering and number of fruits per clusters, fruit breadth and plant height, 10 crosses for number of flowers per cluster, 12 average fruit weight, 3 for number of fruits per plant, 2 number of branches per plant, 6 crosses for fruit length and fruit yield per plant.

No single cross combination exhibited simultaneous significant SCA for all the traits under study. The cross BB $68 \times \mathrm{BB} 45 \mathrm{C}$ was found to show superior SCA effects for days to first flowering (-21.36). This cross involved low $\times$ highgeneral combining ability. Number of flowers per cluster (1.30) and plant height (22.17) was observed highest in the cross $\mathrm{BB}$ $45 \mathrm{C} \times \mathrm{BB} 13$. The cross involved high $\times$ high and low xlow combining parents respectively. Among crosses only one cross combinations BB $26 \times$ BB 68 had shown significant positive SCA effect for number of branches per plant (1.19) involving low $\times$ low parents. BB45C $\times$ BBSR 195-1 was found to show superior SCA effects for fruit length (5.95) and BB $13 \times \mathrm{BB} 44$ for fruit breadth (7.90).This was obtained by crossing high $\times$ high and low $\times$ low general combining lines respectively. Highest SCA effect were observed in BBSR $202 \times$ BB 44 for number of fruits per cluster (1.78) involving low $\times$ high general combiners. The cross BB 68 $\times$ BB67 showed highest SCA effect for average fruit weight (34.59). The combination involved low $\times$ high general combiners.BB $67 \times$ BB $45 \mathrm{C}$ exhibited maximum SCA effects for number of fruits per plant (4.56). The cross were derived from low $\times$ high combining parents. SCA effect for fruit yield per plant (357.69) was found significantly higher for BBSR $202 \times$ BB 13 involving high $\times$ high general combiners. Similar results earlier reported by Shanmugapriya et al., (2009) and Joshi et al., (2008).

In the present study, majority of the crosses exhibited high sca effects involving either low $x$ low, high $\times$ high and low $\times$ high or high $\times$ low general combiners indicating a genetic interaction of the dominance $\times$ dominance, additive $x$ additive, dominance $x$ additive or additive $\times$ dominance type. This has suggested that good cross combinations are not always obtained from high general combiners. The crosses which involved good $\times$ good general combiners includes positive alleles from both the parents and thus can be fixed in the subsequent generations for effective selection of desirable parents if no repulsion phase linkage is present. (Kumar and Kumar, 2017). The high $\times$ low or low $\times$ high crosses, besides exhibiting the favourable additive gca effects of the parent, complement the epistatic effects present in the cross, which would finally result in higher sca effects.(Santhakumar and Salimath, 2013). The crosses involving both the poor combining parents showing high sca must be due to intra and inter allelic interaction. (Shindhe et al., 2011).

The result presented in Table 3 showed that the SCA variances $\left(\sigma^{2} s\right)$ were higher than GCA variances $\left(\sigma^{2} g\right)$ for all the character, indicating preponderance of non-additive gene action in the control of these characters and there is scope for heterosis breeding in brinjal. The importance of variance ratio for gene action in brinjal has also been reported by Aswani and Khandelwal (2005) and Ram and Singh (2007). The data revealed that variance ratio was less than one for all the traits viz., days to first flowering (0.04), plant height (0.13), number of flowers per cluster (0.23), number of branches per plant $(0.10)$, fruit length $(0.22)$, fruit breadth (0.11), number of fruits per cluster (0.12), number of fruits per plant (0.43), average fruit weight (0.12), fruit yield per plant (0.24). Results are conformity with finding of Sao and Meheta (2010) and Suneetha et al., (2008).

From this study, it may be concluded that parent BBSR 202, BB 45C and BB 26 were best general combiners for yield and it contributing traits and yield could be improved by using these parents in hybrid breeding programmes. The specific combination BBSR $202 \times$ BB 13 BB $26 \times$ BBSR 195-1 and BB 45C $\times$ BB 13 could be included for further testing for exploitation of hybrid vigour inbrinjal. The nonadditive gene action in the present study suggested that a breeding procedure, such as 
population improvement (selection) and heterosis breeding can be beneficial for improving yield and its related traits in brinjal.

\section{Acknowledgement}

Experimental facilities and assistance provided by All India Coordinated Research Project on Vegetable Crops, Orissa University of Agriculture and Technology, Bhubaneswar for conductingthe research is duly acknowledged.

\section{References}

Ansari, A.M., and Singh, Y.V. 2014.Combining ability effects for fruit characters in brinjal (Solanummelongena L.).Electronic Journal of Plant Breeding. 5(3):385-393.

Aswini, R.C., and Khandelwal, R.C. 2005. Combining ability studies in brinjal. Indian Journal of Hoticulture.62 (1):3740.

Griffing, J.B., 1956. Concepts of general and specific combining ability in relation to diallel crossing system.Australian Journal of Biological Sciences.9: 463-93.

Joshi, N., Singh, Y.V., and Maurya, S.K. 2008.Combining ability analysis for various quantitative traits in eggplant. (Solanummelongena L.).Annals of Horticulture. 1(1): 32-36.

Kamalakkannan, T., Karuppaiah,P., Sekar,K., and Senthilkumar,P. Line $\times$ Tester analysis in brinjal for yield and shoot and fruit borer tolerance. Indian Journal of Horticulture.64 (4):420-424.

Kumar, R., and Kumar, S.2017. Usefulness of combining ability and gene action studies for parthenocarpicgynocious hybrid development in cucumber. Journal of Hill Agriculture.8 (2): 158-165.
Rai, N., and Asati, B.S.2007.Combining ability and gene action studies for fruit yield and yield contributing traits in brinjal. Indian Journal of Horticulture. 68(2):212-215.

Ram, K., and Singh, P. 2007.Combining ability and gene effect for yield and quality characters in brinjal (Solanummelongena L.).Indian Journal of Genetics.67 (1):93:94.

Shanmugapriya, P., Ramya, K., and Senthilkumar, N. 2009. Studies on combining ability and heterosis for yeld and growth parameters in brinjal. $\begin{array}{lll}\text { (Solanummelongena } & \text { L.). } & \text { Crop }\end{array}$ Improvement.36 (1):68-72.

Shanthakumar, S.A.G., and Salimath, P.M. 2013. Selection of parents based on combining ability studies in okra (Abelmoschusesculentus (L.) Moench. Karnataka Journal of Agricultural Science. 26: 6-9.

Shindhe, K.G., Bhalekar, M.N., and Patil, B.T., 2011.Combining ability of quantitative characters in brinjal.38 (2): 231-234.

Suneetha, Y., Kathiria, K.B., Patel, J.S., and Srinivas, T., 2008. Studies on heterosis and combining ability in late summer brinjal. Indian Journal of Agriculture Research. 42(3):171-176.

Thangavel, P., 2011. Studies on gene action and combining ability for yield and other quantitative traits in Brinjal (Solanummelongena L.).International Journal of Current Agricultural Sciences. 2(1):23-25.

Venkata Naresh, B., Dubey, A.K., Tiwari, P.K., and Dabbas, M.R. 2014. Line $\times$ Tester analysis for yield components and cercospora leaf spot resistance in brinjal (Solanummelongena L.). Electronic Journal of Plant Breeding. 5(2):230-235.

\section{How to cite this article:}

Madhusmita Dishri and Mishra, H.N. 2017. Estimation of Combining Ability and Gene Action Studies in Brinjal (Solanum melongena L.). Int.J.Curr.Microbiol.App.Sci. 6(10): 3584-3591. doi: https://doi.org/10.20546/ijcmas.2017.610.422 